\title{
A hybrid visual-model based robot control strategy for micro ground robots
}

\author{
Cheng $\mathrm{Hu}^{1[0000-0002-1177-2167]}$, Qinbing $\mathrm{Fu}^{1[0000-0002-5726-6956]}$, \\ Tian Liu ${ }^{1[0000-0002-9445-1878]}$, and Shigang Yue $1[0000-0002-1899-6307]$ \\ University of Lincoln, Lincoln, UK, \\ $\{\mathrm{chu}$, qifu, tliu, syue $\}$ lincoln.ac.uk
}

\begin{abstract}
This paper proposed a hybrid vision-based robot control strategy for micro ground robots by mediating two vision models from mixed categories: a bio-inspired collision avoidance model and a segmentation based target following model. The implemented model coordination strategy is described as a probabilistic model using finite state machine (FSM) that allows the robot to switch behaviours adapting to the acquired visual information. Experiments demonstrated the stability and convergence of the embedded hybrid system by real robots, including the studying of collective behaviour by a swarm of such robots with environment mediation. This research enables micro robots to run visual models with more complexity. Moreover, it showed the possibility to realize aggregation behaviour on micro robots by utilizing vision as the only sensing modality from non-omnidirectional cameras.
\end{abstract}

Keywords: micro robot, visual model, bio-inspired, collision avoidance, image processing

\section{Introduction}

Bio-plausible visual models inspired from insects are getting importance in robotics for various of visual-motor tasks, such as the trajectory stabilization and navigation task inspired by Elementary Motion Detector (EMD) [1], collision avoidance (CA) inspired by Lobular Giant Movement Detector (LGMD) [2,3], homing task inspired by the mushroombody of ants [4] and also navigation in spired by Small Target Motion Detectors (STMD) [5], by taking advantage of their reliability in coping with rapid changing scenarios with only minimum amount of neurons occupied. Comparing to conventional reactive navigation algorithms based on computer vision techniques such as standard optic flow [6], these bio-inspired models are usually free from massive calculation such as object recognition or distance estimation. Benefiting from the simplicity and efficiency, they are especially feasible for micro robots that often have only limited computational power on-board $[7,8]$.

Take collision avoidance as one example, the neural structure LGMD which located in the locust's optic lobe is believed to be responsible for detecting looming objects and triggering escaping maneuver [9]. For decades, it has 
inspired researchers to implement the neural network into computational models for autonomous vehicles. The computational model of LGMD such as the Embedded-LGMD (ELGMD) [8] has been proved to be reliable in dealing with collision situations in dynamic scenarios. By producing neural spikes only when the imminent collision is rapid and close enough, the LGMD is well-recognized by its high non-linearity [10] and directional selectivity, which is different from other bio-inspired visual models $[1,11]$.

As a result, it will be interesting to investigate the possibility and performance of utilizing both LGMD-based collision avoidance model and visual navigation models to perform more complex tasks. Since they usually hold contradictory purposes, when both collision avoidance and visual navigation models are utilized, their feature have to be carefully mediated to avoid conflicting motion commands. Among various of approaches at higher level to integrate these visual models, such as feedback linearisation [12], fuzzy logic [13] and finite state machine (FSM) [14], the FSM is one of the most favoured approach on micro robot platforms due to its predictability responding to inputs without altering the performance of sub-models.

This paper proposed an approach of realizing a hybrid visual-motor control strategy for micro robots, allowing them to accomplish multiple tasks with certain goal. The proposed strategy which can be described as probabilistic model using FSM is composed of visual models from two different categories: the ELGMD model and a visual navigation approach based on image segmentation to imitate the target following behaviour. The stability and convergence of this robot control strategy is demonstrated by a series of experiments, including aggregation behaviour from a swarm of low-cost micro ground robots named Colias $I V$. To the best of the authors knowledge, it is the first attempt of utilizing a common camera as the only sensor, and processing image information to implement aggregation behaviour without communication on real micro robots.

The rest of this paper are organized as follows: section 2 describes the proposed algorithms implemented in individual robots; section 3 illustrates a series of experiments to test the performance of proposed robot system and the swarm scenario; we conclude this research in section 4 .

\section{Models and Methods}

\subsection{The Bio-inspired Collision Detection Model}

Avoiding collisions is always crucial for autonomous robots. In this study, the ELGMD is deployed to serve CA purpose. As demonstrated in previous studies $[8,15]$, even this feature alone, an individual robot is able to establish autonomous motion inside a constrained arena.

The ELGMD is a layered neural model formed by five layers with lateral inhibition mechanism and two single cells. The computational model contains only low-level image processing such as excitation transferring and neighbouring operations. The ELGMD model predicts an imminent collision by abstracting 


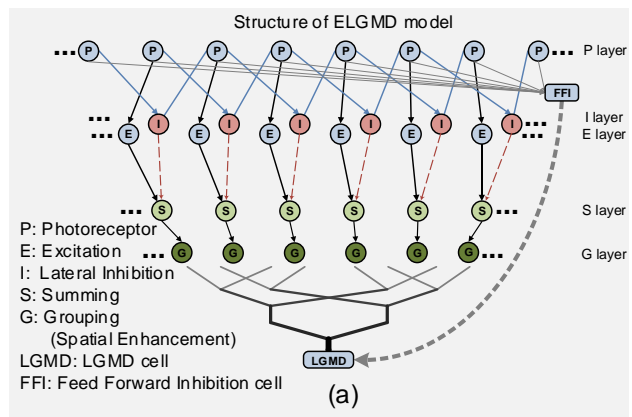

(a)

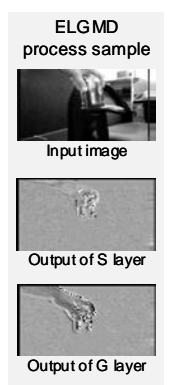

(b)

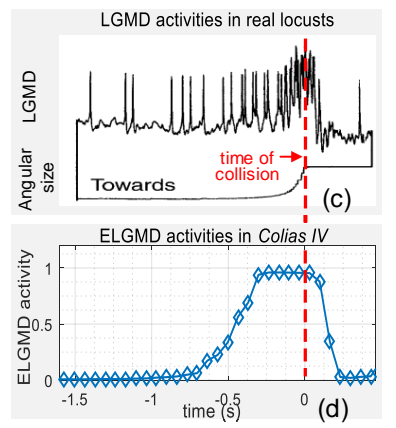

Fig. 1: The illustration of the implemented computation model ELGMD for CA. (a) The ELGMD model's architecture. The input of the $P$ layer are the luminance change. Inhibitions (The $I$ layer and the FFI cell) are indicated with dotted lines and have one frame delay. Excitations are indicated with solid lines which have no delay. (b) Example of ELGMD process. The output of $S$ layer filters out the stationary background, and the output of $G$ layer enhances the edges of moving foreground; (c) Intracellular recordings from the LGMD of a real locust viewing an approaching object (figure adapted from [9] with permission). (d) Output of ELGMD tested with a similar configuration comparing to (c), showing the increasing LGMD activity before collision.

Table 1: Robot Control Strategy Definition by ELGMD model

\begin{tabular}{|c|c|c|c|}
\hline $\begin{array}{l}\text { Neuron } \\
C_{f}^{L G M D}\end{array}$ & $\begin{array}{l}\text { Status } \\
C_{f}^{F F I}\end{array}$ & Decision & Action \\
\hline 0 & 0 & No collision & Wander $(60 \mathrm{~mm} / \mathrm{s})$ \\
\hline 1 & 0 & Collision & Turn $\left(180 \pm 60^{\circ}\right)$ \\
\hline $\mathrm{X}$ (any) & 1 & Sudden Change & Stop for a while $(0.3 s)$ \\
\hline
\end{tabular}

the visual motion information, i.e. the fast expanding profile of approaching objects into the activity level of LGMD cell, which is further transformed into neural spikes according to a fixed threshold. Confirmation of impending collision is generated by successive LGMD spikes. In some circumstances such as self turning, false spikes caused by sudden motion in vision could be suppressed by the feed-forward inhibition cell (FFI), which is also an activity from visual motion. The explanation of ELGMD and its performance are illustrated in figure 1. In the Colias IV robot, the process of ELGMD takes the full size (99x72) of captured image into calculation. For the robot's motion settings, since the speed characteristics is not the primary goal to study in this paper, the motion speed is not set to the maximum, but to the power-efficient range.

In consistency with ELGMD models utilized in autonomous CA experiments, the parameters and controlling logic are kept as previously chosen [8]. The robot's motion is controlled according to the status of ELGMD model, determined by both of the LGMD and FFI cells' activity, which are listed in table 1. When no collision is detected, the robot is allowed to wander forward without constraints. To keep the system as simple as possible, the collision avoiding behaviour is defined by a in-position turning to another direction, which is almost an U- 


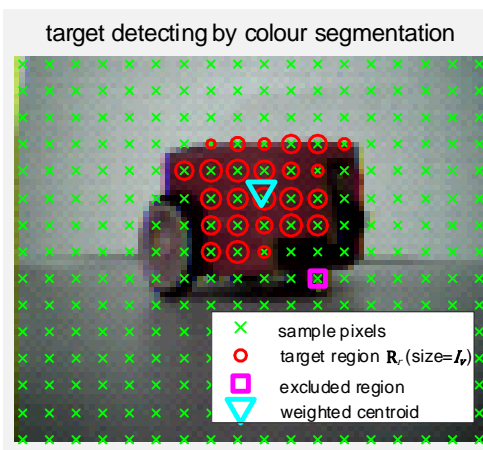

(a)

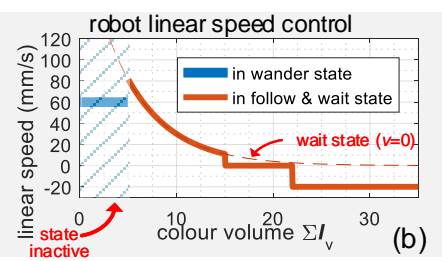

robot turning speed control (in follow state)

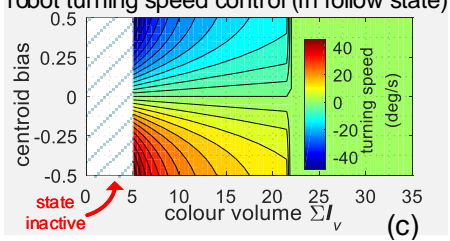

Fig. 2: Illustration of the target detection and following model. (a) The target detection, showing with the target robot staying $9 \mathrm{~cm}$ away. The red circle indicates the points belongs to the target, while the colour intensity is represented by the size of circle; (b) The robot's linear motion speed control behaviour. (c) The robot's rotation motion control by both target volume and centroid bias. In (b) and (c), when target volume is less than 5 , the model is inactive which is overtaken by the CA model.

turn with randomly generated margin $\left(180 \pm 60^{\circ}\right)$ to prevent deadlocking in case trapped in the corner or several obstacles. When a large object is translating in front of the robot that producing spikes to the FFI cell, the robot will stop for a while until safe.

\subsection{Vision cue-based Target Following Model}

Another elementary part of this hybrid vision model is to track and follow an object, either a partner robot, or an anchor position, so that multiple robots could gather together and form an accumulating group. As one example of simple conventional visual model, it contains two parts: a light-weight visual target detection algorithm to identify the object, and a robot navigation method to follow the object continuously. Example of this process is shown in figure 2.

Visual target detection: The visual target detection algorithm is mainly based on the target's colour property. In this colour segmentation approach, we have chosen red as the only interested colour since it has the highest saturation response, which could be observed by the robot from far away.

Respecting to the robot platform's computational power, the algorithm is kept as simple as possible. The input image $\boldsymbol{I}$ used for this model has been down-sampled to $18 \times 15$ pixels to balance the image quality and the CPU\&RAM occupation. The colour space is transformed into hue-saturation-value (HSV) [16] from it's original YUV format, thus majority of required chromatic cue is represented by the hue value. In HSV colour space, each pixel $\boldsymbol{I}_{(i, j)}$ in the image $\boldsymbol{I}$ is represented by the values in three channels:

$$
\boldsymbol{I}(i, j)=\left[\boldsymbol{I}_{h}(i, j), \boldsymbol{I}_{s}(i, j), \boldsymbol{I}_{v}(i, j)\right]^{\prime}
$$


At first, the down-sampling image is passes through a hue-saturation mask to match certain conditions. To prevent the hue adrift problem and to increase the robustness against different illumination conditions, suspicious pixels that belongs to the target are checked by the hue range and minimum saturation:

$$
\boldsymbol{M}_{\text {red }}(i, j)= \begin{cases}\text { true } & \text { if }\left(-10^{\circ} \leq \boldsymbol{I}_{h(i, j)} \leq-10^{\circ}\right) \bigcap\left(\boldsymbol{I}_{s(i, j)} \geq 0.3\right) \\ \text { false } & \text { otherwise }\end{cases}
$$

where $\boldsymbol{M}_{\text {red }}(i, j)$ is the suspicious region. The fixed thresholds are chosen empirically. Followed by this, all suspicious regions are joined and analysed by 4connection to find the correct target region. Only the largest region $\mathbf{R}_{r}$ is treated the correct target area, excluding the others. Once a red object is identified by above procedures, two metrics are measured for the motion generation procedure: 1) the identified target's colour volume $D_{o b j}$ (or the "mass" of target region), which is the zero-order moment of target region's value channel:

$$
D_{o b j}=\mu_{(0,0)}^{\mathbf{R}_{r}}=\sum_{i, j \in \mathbf{R}_{r}} \boldsymbol{I}_{v}(i, j)
$$

and 2) the bias $B_{o b j}$ of the object's centroid against the image centre, which is the normalized horizontal first-order moment:

$$
B_{o b j}=\frac{\mu_{(1,0)}^{\mathbf{R}_{r}}}{\mu_{(0,0)}^{\mathbf{R}_{r}}}=\frac{\sum_{i, j \in \mathbf{R}_{r}} \boldsymbol{I}_{v}(i, j) \cdot\left(j-\frac{j_{\max }}{2}\right)}{\sum_{i, j \in \mathbf{R}_{r}} \boldsymbol{I}_{v}(i, j) \cdot j_{\max }}
$$

in which $j$ is the horizontal index of a pixel, and $j_{\max }$ is the width of the image.

Motion control: The goal of motion control is to drive a robot towards the target to a region, where the target is in front of the follower with a certain distance. Specifically, this behaviour contains three phases:

P1: If the target emerges from far away, the robot accelerates to charge towards it, until the projected image has reached a certain size then becomes phase 3. The motion speed decrease exponentially during approach,

P2: If the target appears suddenly or becomes too close that contributes to a large region of image, which could happen when the target is moving, the robot retracts to leave enough space to enter P3.

P3: A safe zone is set when the size of target is satisfactory, then the robot enters the wait state. During this period, the robot only tunes its orientation slightly towards the centre of object but no displacement is made.

These phases can be described as functions of two group of variables: the robot's linear speed $v$ and rotation speed $\omega$ controlled by the calculated metrics from image processing $D_{o b j}$ and $B_{o b j}$, as illustrated in figure 2(b) and 2(c).

Notice that, by utilizing this visual navigation model alone, the initial catch up speed have to be greater than the target object, which could be another robot, in order to make this system converge [12].

\subsection{The Hybrid Robot Control Strategy}

The hybrid robot control strategy is based on the probabilistic approach: the finite state machine (FSM), since for such two visual models that generate 


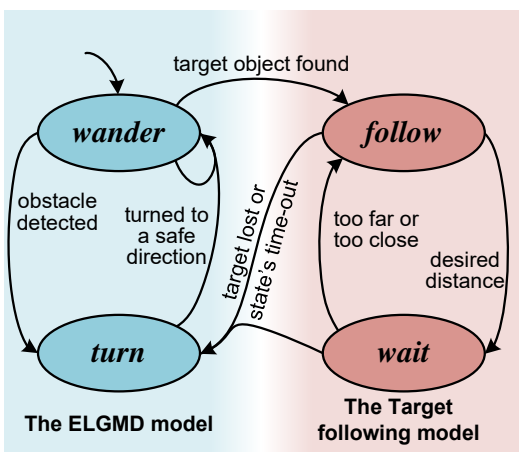

(a)

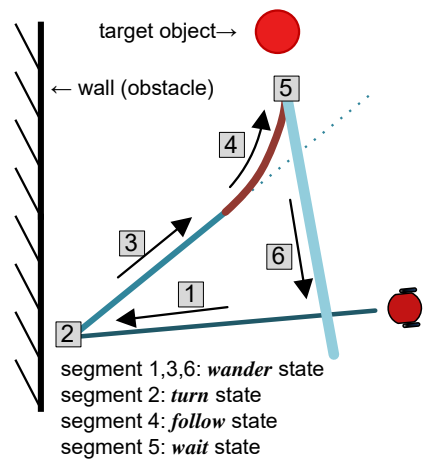

(b)

Fig. 3: The proposed finite state machine to incorporate the two visual models. (a) The illustration of proposed FSM, whose initial state is the wander state. (b) The illustration of a typical scenario controlled by this hybrid visual system.

conflicting behaviours, actions from only one model can be taken by the robot at a time according to its current situation, in order to ensure stability and consistency. In total, there are four behaviours generated by two models that need to be controlled, which are the wander / turn generated by the ELGMD model, and the follow / wait generated by the target following model. The structure and an example of robot behaviour are depicted in figure 3 .

In this FSM logic, the wander state is set to be the initial and default state, while the other states are restricted with conditions. In this way, suppose a robot inside an arena surrounded by walls, it would perform such a behaviour:

1. The robot wanders within the arena to establish autonomous navigation as long as possible, by trying to avoid collisions against walls or any other unidentified object by accepting motion commands from the ELGMD model.

2. When a target object is encountered, the robot enters the follow state to maintain certain distance behind. If the target object is stationary, the follower stops in the wait state. A timer is set once the motion commands from the target following model is activated. The activity last for a certain time $t_{w}$ before it rotates to another direction, in order to return to the wander state to continue exploring the arena.

\subsection{The Robot Platform and Experiment Arena Configuration}

In this research, the micro robot platform Colias IV is utilized [17]. Colias IV is a micro ground robot occupying a circular footprint bearing the diameter of $4 \mathrm{~cm}$. The motion is provided by two differential driven wheels. The Colias $I V$ is equipped with a strong ARM-Cortex M4 micro-controller running at $180 \mathrm{MHz}$ with embedded SRAM of 256 Kbytes, which ensures the required computation power for both proposed image processing tasks. The images used in the visual tasks are captured continuously by a tiny CMOS camera on-board with resolution configured at 99x72, 30 frames per second. With regarding to the 
frame rate, the system is working in real-time mode that each process has certain time allowance. In the total frame duration of $33 \mathrm{~ms}$, the ELGMD need $7 \pm 2 \mathrm{~ms}$ to process, the target finding model occupies $11 \pm 1 \mathrm{~ms}$, and the rest process use up to $1 \mathrm{~ms}$. The robot in the experiments are coated with a 3D-printed red shell, in order to be distinguishable by the target following model, as shown in figure $4(\mathrm{a})$.

The experiments are conducted in an arena sizing $175 \times 155 \mathrm{~cm}$, surrounded by wall of $15 \mathrm{~cm}$ high and decorated by wall papers with black\& white patterns. The floor of the arena is covered by black tiles. There are two types of illumination cast into this arena to separate two zones by brightness levels. The bright zone is irradiated by four LED spot lights above the arena, while the dark zone is lighted by ambient lamps of the whole room. Both light temperature has been tuned to $4000 \mathrm{~K}$ for consistency. Equipped with SORAA SNAP systems, the light beams produced by the LED have straight and sharp edges, leaving the transition border with thickness of almost $5 \mathrm{~cm}$. Two red cue balls with diameter of $\phi=48 \mathrm{~cm}$ are fixed in the arena, serving as static stimuli. One cue ball is placed in centre of the bright zone and the other one in the dark zone.

Robots behaviour were recorded by overhead camera. Their trajectories are tracked and analysis by whycon system [18] by utilizing the circle markers on top of the robot [19], as shown in figure 4 .

\section{Experiments and Results}

\subsection{Individual Robot Performance Tests}

The performance of hybrid visual model on the individual robot is tested accordingly. All tests in this subsection is done in the bright zone. Results of the data was illustrated in figure 5 .

First, the stability of proposed visual models are tested by challenging the robot with static stimuli, as illustrated in figure 5a.The following metrics are analysed and each scenario is repeated for 50 times:

1. the success rate of collision detection and avoidance $\eta_{c}$;

2. the Distance to Collision (DTC) of the ELGMD model for both the wall obstacle $d_{t c}^{w a l l}$ and a green ball $d_{t c}^{\text {ball }}$ for comparison, which is the distance between the robot and the obstacle when it turns away;

3. the ratio of switching models in a competitive scenario $P_{t}$, which is defined by: when two objects are placed in front of the robot, the ratio of switching from wander to follow and from wander to turn;

4. the distance where the robot stops in front of a red object $d_{a t t}(1)$, or two objects $d_{a t t}(2)$.

Results of these tests are concluded in table 2. The results have demonstrated that the visual models work stable to realize their own goals. Moreover, it reveals that, for the visual target following model, the sensitivity and range increases as the target cluster expands, since a larger region of targets contributes to more obvious visual cues to be identified from far away. 


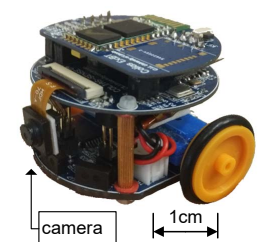

(a)

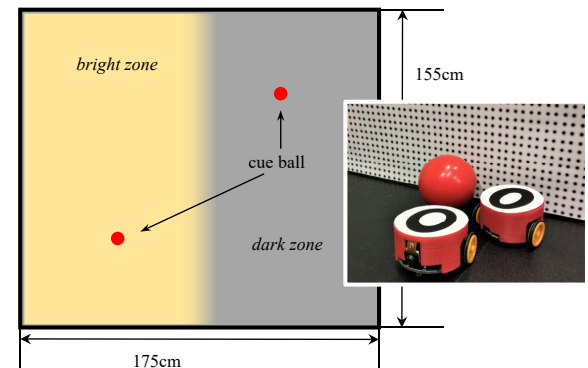

(b)

Fig. 4: The robot platform and experiment arena configuration. (a) The Colias IV robot deployed in this research. (b) The experiment arena set-up, showing the photo of a cue ball and Colias $I V$ covered by $3 \mathrm{D}$ printed shells.

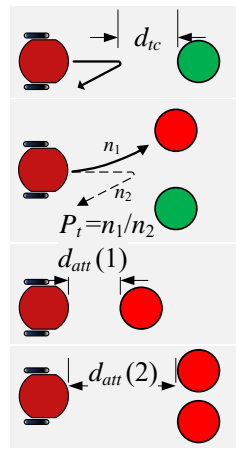

(a)
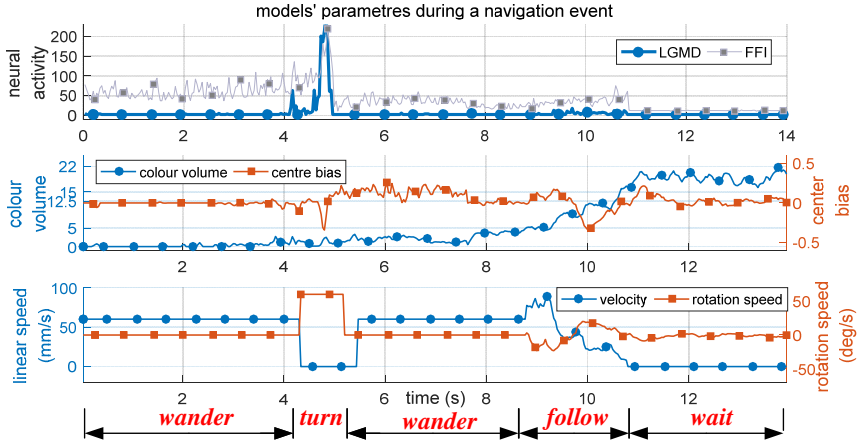

(b)

Fig. 5: The tests of hybrid model on individual robot. (a) Schematic of measuring the metrics of the hybrid model. The results are shown in table 2 ; (b) One piece of the variables of a running robot during an arena test. The robot's trajectory is similar to which of figure 3(b). The robot's states are indicated at the bottom of plots.

Then, the variables of a robot deployed inside the arena are logged and analysed to show the dynamics of the visual models. For a typical scenario where the robot's trajectory is similar to figure $3(\mathrm{~b})$, the data are plotted in figure 5b. The recorded results show that the ELGMD has evoked a turning action when a collision is detected at around $4.5 \mathrm{~s}$. Then at $8.3 \mathrm{~s}$, a target object has been identified thus attracts the robot to charge towards it. The orientation is tuned continuously. Finally at $10.8 \mathrm{~s}$, the robot stops behind the object. The series of states switching meet our expectations.

\subsection{Robot Aggregation Tests}

Since the robots can join and leave the cluster at any time, one typical approach to study the stability of aggregation which is dynamically changing is to study 
Table 2: The Test Results of Individual Robot Behaviours

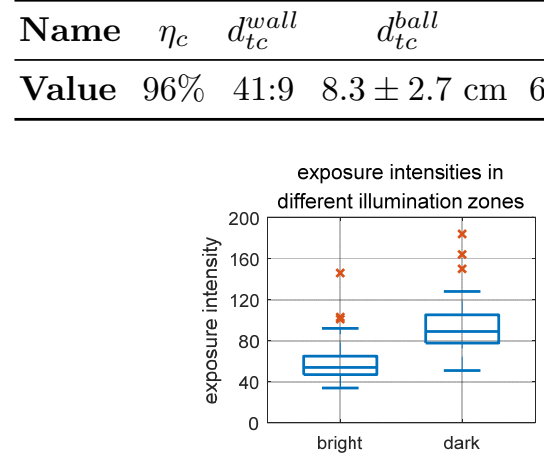

(a)

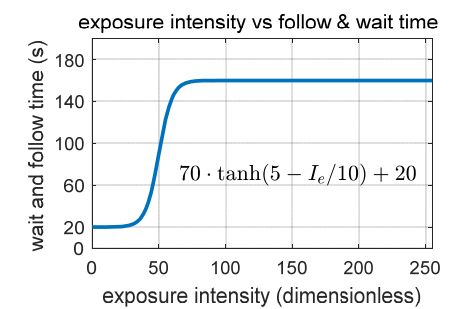

(b)

Fig. 6: (a)The tested exposure intensities under different illumination status; (b) The curve to tune wait time $t_{w}$ by exposure intensity $I_{e}$.

the discriminative cluster size controlled by environment. By adjusting the time that each robot remains in the cluster, i.e. the $t_{w}$, it is obvious that the longer time each robot stays inside a cluster, the larger size.

Being inspired by the aggregation behaviour found in honeybees and cockroaches [20], we employ the ambient illumination as the controlling factor, which is embodied by the camera's exposure intensity $I_{e} \in[0,255]$, an integer automatically adjusted in the camera by a build-in histogram-based algorithm. Thus no additional sensors or communication methods are required. Its intention is to maintain the brightness level of captured images. Therefore, the value is tightly tuned by the view of the robot.

One test on the relationship between $I_{e}$ and the robot's vision is illustrated in figure 6 . The results showed that a worse illumination condition would be compensated by a higher exposure intensity, and vice versa. By using this mechanism, the $I_{e}$ then tunes the robot's $t_{w}$ by:

$$
t_{w}=70 \cdot \tanh \left(5-\frac{I_{e}}{10}\right)+20
$$

The Aggregation Experiments: The experiments are conducted with different population sizes, given by 7,11 and 15 robot agents respectively. Each experiment last for 15 minutes. At the beginning the robots were placed randomly inside. Aggregation number of both bright zone and dark zone were recorded and logged. Since there is no complete stop condition for any robot, the formation of groups are always fluctuating. This doesn't interfere us to analyse their properties by comparing the group sizes along time. For all three trials, we investigated the largest cluster in both zones along time. Records and results of the experiments are redrawn in figure 7 .

Results: Result of the experiments show that robots tend to aggregate at darker zones rather than bright zones regardless of group sizes. For each group size, the 


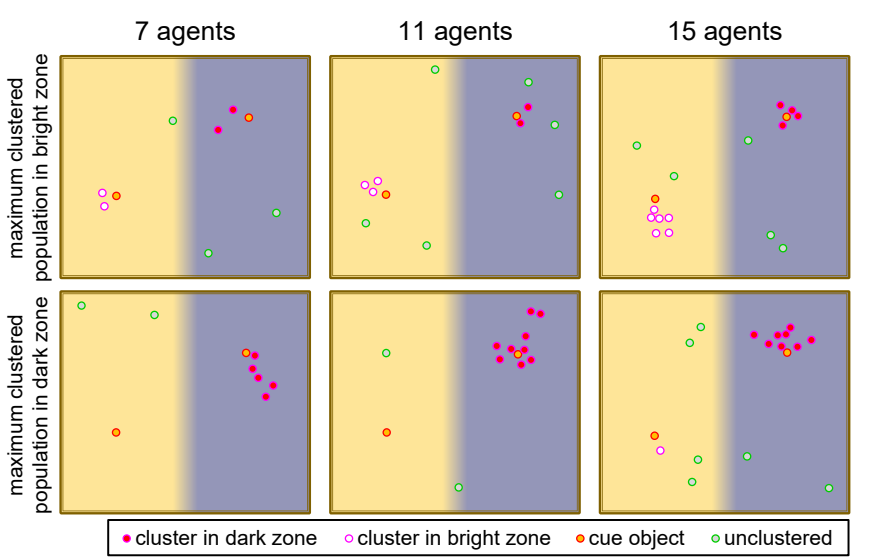

(a)

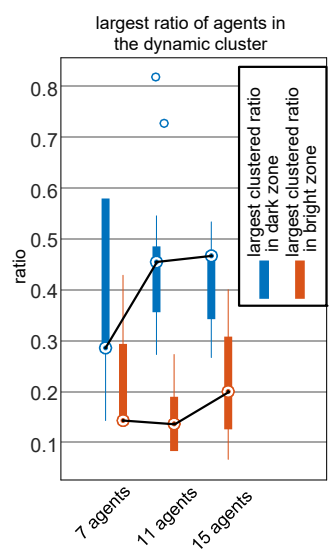

(b)

Fig. 7: The illustration of arena experiments results with multiple robots when the largest clustered population has reached in either zones (see scales in figure 4b); (b) The largest cluster population distributions in all experiment trials.

dark zone always restrain robots for longer thus there are higher chances for more robots to meet and stop. On the other hand, the cluster in the bright zone vanishes quickly due to the short $t_{w}$. As a main factor that cause the environment selective behaviour, this dynamic behaviour is one of the common point in probabilistic aggregation. This behaviour is also found in the cockroaches inspired robots [20,21], whose chance to join or leave a cluster is affected by both the cluster size and environment darkness.

\subsection{Discussion}

Micro robots with strong vision ability have shown its potential in swarm robotics, but the approaches are not always straightforward. Being different from the utilization of conventional sensors such as sound, light or infra-red sensors [22], information from images is highly sensitive to the relative motion of target and viewing angle [23]. In earlier researches, some approaches use additional information [23] such as infra-red sensors to identify surrounding environment and prevent collisions, while some employ an omnidirectional camera [12] to expand viewing angle.

Although the vision has shown its potential in acting as the primary sensor to achieve multi-tasking [24], care should be taken at designing to get stable outputs for before motion controlling. Since the robots can only detect objects only in view, they cannot react to objects in other directions [5], thus aggregation is less likely to trigger than those robots equipped with omnidirectional sensors [22]. The cue balls act as static stimulus in the arena. They provide stable signals which help robots to gather around faster. As the population size gets larger, there is a higher chance that two robots run in a face to face trajectory, which causing a group without the help from cue balls. 


\section{Conclusion}

The paper has discussed an approach to mediate two visual tasks from different categories and even with conflicting purposes in micro robots to serve multipurpose tasks. This hybrid visual coordination model is described by a FSM that activates either model according to the robot's situation thus no interference is introduced to each other. The two visual models are a bio-inspired collision detection neural model, and a colour-sensitive target following algorithm. Both vision systems are computationally efficient and stable to be implementation on a microprocessor. The proposed hybrid model is realized on the micro robots named Colias $I V$ to demonstrate the robustness.

Systematic experiments showed that the bio-inspired collision avoidance model ELGMD can cooperate with other visual models to achieve multi-tasks. The proposed hybrid visual model is feasible to be deployed in low-cost micro robots especially for educational purposes, which enables the collective behaviour study by micro robots utilizing normal camera as the only sensor.

In the future, study will be conducted on 1) to further investigate the aggregation behaviour evoked by visual inputs in swarm scenarios; 2) to improve the proposed target following models to achieve better precision and sensitivity.

\section{ACKNOWLEDGEMENT}

This work is supported by EU-FP7-IRSES project HAZCEPT(318907) and Horizon2020 project STEP2DYNA.

\section{References}

1. J.-C. Zufferey and D. Floreano, "Toward 30-gram autonomous indoor aircraft: Vision-based obstacle avoidance and altitude control," in Robotics and Automation, (ICRA) IEEE International Conference on, 2005, pp. 2594-2599.

2. H. G. Meyer, O. Bertrand, J. Paskarbeit, J. P. Lindemann, A. Schneider, and M. Egelhaaf, "A bio-inspired model for visual collision avoidance on a hexapod walking robot," in Biomimetic and Biohybrid Systems: 5th International Conference, Living Machines 2016, Proceedings, 2016.

3. Q. Fu, C. Hu, T. Liu, and S. Yue, "Collision selective lgmds neuron models research benefits from a vision-based autonomous micro robot," in 2017 IEEE/RSJ International Conference on Intelligent Robots and Systems, 2017, pp. 3996-4002.

4. P. Ardin, F. Peng, M. Mangan, K. Lagogiannis, and B. Webb, "Using an insect mushroom body circuit to encode route memory in complex natural environments," PLoS computational biology, vol. 12, no. 2, p. e1004683, 2016.

5. Z. Bagheri, B. Cazzolato, S. Grainger, D. O'Carroll, and S. Wiederman, "An autonomous robot inspired by insect neurophysiology pursues moving features in natural environments." Journal of neural engineering, vol. 14, no. 4, 2017.

6. J. Serres and F. Ruffier, "Optic flow-based robotics," Wiley Encyclopedia of Electrical and Electronics Engineering, 2016.

7. J.-C. Zufferey and D. Floreano, "Fly-inspired visual steering of an ultralight indoor aircraft," IEEE Transactions on Robotics, vol. 22, no. 1, pp. 137-146, 2006. 
8. C. Hu, F. Arvin, C. Xiong, and S. Yue, "Bio-inspired embedded vision system for autonomous micro-robots: The lgmd case," IEEE Transactions on Cognitive and Developmental Systems, vol. 9, no. 3, pp. 241-254, Sept 2017.

9. F. C. Rind and P. J. Simmons, "Seeing what is coming: building collision-sensitive neurones," Trends in Neurosciences, vol. 22, no. 5, pp. 215-220, 1999.

10. S. B. i. Badia, P. Pyk, and P. F. M. J. Verschure, "A fly-locust based neuronal control system applied to an unmanned aerial vehicle: the invertebrate neuronal principles for course stabilization, altitude control and collision avoidance," The International Journal of Robotics Research, vol. 26, no. 7, pp. 759-772, 2007.

11. N. Franceschini, J.-M. Pichon, and C. Blanes, "From insect vision to robot vision," Phil. Trans. R. Soc. Lond. B, vol. 337, no. 1281, pp. 283-294, 1992.

12. A. K. Das, R. Fierro, V. Kumar, J. P. Ostrowski, J. Spletzer, and C. J. Taylor, "A vision-based formation control framework," IEEE transactions on robotics and automation, vol. 18, no. 5, pp. 813-825, 2002.

13. P. Benavidez and M. Jamshidi, "Mobile robot navigation and target tracking system," in System of Systems Engineering (SoSE), 2011 6th International Conference on. IEEE, 2011, pp. 299-304.

14. F. Arvin, A. E. Turgut, F. Bazyari, K. B. Arikan, N. Bellotto, and S. Yue, "Cue-based aggregation with a mobile robot swarm: a novel fuzzy-based method," Adaptive Behavior, vol. 22, no. 3, pp. 189-206, 2014.

15. S. B. i Badia, U. Bernardet, and P. F. Verschure, "Non-linear neuronal responses as an emergent property of afferent networks: a case study of the locust lobula giant movement detector," PLoS computational biology, vol. 6, no. 3, 2010.

16. M. Loesdau, S. Chabrier, and A. Gabillon, "Hue and saturation in the rgb color space," in International Conference on Image and Signal Processing. Springer, 2014, pp. 203-212.

17. C. Hu, Q. Fu, and S. Yue, "Colias iv: the affordable micro robot platform with bioinspired vision," in 19th Towards Autonomous Robotic Systems (TAROS), 2018.

18. T. Krajník, M. Nitsche, J. Faigl, P. Vaněk, M. Saska, L. Přeučil, T. Duckett, and M. Mejail, "A practical multirobot localization system," Journal of Intelligent ES Robotic Systems, vol. 76, no. 3-4, pp. 539-562, 2014.

19. P. Lightbody, T. Krajník, and M. Hanheide, "A versatile high-performance visual fiducial marker detection system with scalable identity encoding," in Proceedings of the Symposium on Applied Computing. ACM, 2017, pp. 276-282.

20. N. Correll and A. Martinoli, "Modeling self-organized aggregation in a swarm of miniature robots," in IEEE 2007 International Conference on Robotics and Automation Workshop on Collective Behaviors inspired by Biological and Biochemical Systems, no. SWIS-CONF-2007-002, 2007.

21. S. Garnier, J. Gautrais, M. Asadpour, C. Jost, and G. Theraulaz, "Self-organized aggregation triggers collective decision making in a group of cockroach-like robots," Adaptive Behavior, vol. 17, no. 2, pp. 109-133, 2009.

22. S. Kernbach, D. Häbe, O. Kernbach, R. Thenius, G. Radspieler, T. Kimura, and T. Schmickl, "Adaptive collective decision-making in limited robot swarms without communication," The International Journal of Robotics Research, vol. 32, no. 1, pp. $35-55,2013$.

23. M. Gauci, J. Chen, W. Li, T. J. Dodd, and R. Groß, "Self-organized aggregation without computation," The International Journal of Robotics Research, vol. 33, no. 8, pp. 1145-1161, 2014.

24. A. Denuelle and M. V. Srinivasan, "Bio-inspired visual guidance: From insect homing to uas navigation," in Robotics and Biomimetics (ROBIO), 2015 IEEE International Conference on. IEEE, 2015, pp. 326-332. 